\title{
Editorial
}

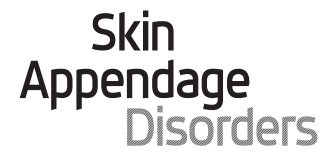

Skin Disorders
Skin Appendage Disord 2018;4:201

DOI: $10.1159 / 000488440$
Published online: April 10, 2018

\section{Is Biotin Safe for Dermatology Patients?}

In the 3rd volume of 2017 of this journal [Skin Appendage Disord 2017;3:166-169], Patel and colleagues present an excellent review of the literature concerning the use of biotin for hair loss. Their conclusions state: "Though its use as a hair and nail growth supplement is prevalent, research demonstrating the efficacy of biotin is limited." Further, they conclude that "... there is lack of sufficient evidence for supplementation in healthy individuals."

On November 28, 2017, the US FDA issued an alert for the public, health care providers, laboratory personnel, and laboratory test developers, stating that biotin, also called vitamin $\mathrm{B} 7$ or vitamin $\mathrm{H}$, if administrated in high doses, can falsely alter several test results that use biotin technology by indicating either too high or too low values. Several hormonal tests and, most importantly, tests for cardiac health used in cardiac infarction, such as troponin, are affected by this problem. In addition, the FDA has seen a report on the death of a patient taking high doses of biotin due to falsely low troponin test results related to biotin interference with this laboratory test.

Following this issue, Roche, a pharmaceutical company that develops immunoassay tests including the Troponin T Gen 5 assay, supports the recommendation issued by the FDA and states that "... at least $8 \mathrm{~h}$ wait time before blood draw for a Roche immunoassay test is recommended" after high doses of biotin administration.

As dermatologists are prescribers of biotin in high doses, they should be aware firstly of the probable lack of any efficacy in hair or nail growth disorders, or that in any case, additional studies are needed before a final result is stated, and secondly that the daily recommended dosage of biotin in otherwise healthy patients should not exceed $0.03 \mathrm{mg} /$ day. At this level, it does not interfere with any performed laboratory tests. Supplements marketed for hair, nail, or skin growth disorders might contain up to $20 \mathrm{mg}$ of biotin, which is more than 650 times the recommended daily dosage.

In any case, dermatologists should inform their patients that after high doses of biotin, they should wait at least $8 \mathrm{~h}$ before any blood is drawn for laboratory examinations and that they should inform healthcare providers about all multivitamins or food supplements they are on, as these might contain biotin in high doses.

Dimitris Rigopoulos Boni Elewski Antonella Tosti 